Int. J. Speleol. 10 (1978), pp. $269-277$

\title{
Synthèse bibliographique sur la toxicité des substances fluorescentes utilisées en Hydrologie (1)
}

\author{
par
}

\section{J. MOLINARI (2) et J. ROCHAT (3)}

A bibliographical Synthesis on the toxicity of fluorescent substances used in Hydrology.

\section{SUMMARY}

This synthesis has been established from three recent studies and from a complementary research bibliography.

It draws togetter the names of fluorescent substances actually used or experimented with as tracers in hydrology and draws attention to their uses and relative problems in their uses. The adjoining annex gives an exhaustive list of publications received on the subject (35 titles).

\section{RAPPEL SUR LES DIFFERENTS MODES D'APPROCHE D'UNE ETUDE TOXICOLOGIQUE}

\subsection{Interactions de la substance à étudier avec le milieu.}

Bien que toute étude toxicologique doive débuter par un examen de l'action sur les êtres vivants de la substance considérée isolément, il serait nécessaire, pour mener à bien une étude de toxicité approfondie, de tenir compte des interactions de la substance avec le milieu, interactions qui peuvent se révéler bénéfiques grâce à l'existence d'antagonismes entre substances, soit au contraire néfastes par l'aggravation des risques sous l'effet de phénomènes de synergies. On devine que ce second stade d'investigation, dont l'ampleur n'a pas de limites si l'on tient à prendre en considération ses intrications avec la chaine écologique, ne peut être envisagé que pour des substances dont l'élaboration et l'usage soulèvent des problèmes cruciaux, et il fallait s'attendre à ce qu'il n'ait encore jamais été abordé pour les substances qui nous intéressent.

(1) Synthèse rédigée en juin 1977 dans le cadre des activités du Groupe des Applications de la Radioactivité et des Traceurs en Hydrologie et dans l'Industrie, au Centre d'Etudes Nucléaires de Grenoble du Commissariat à l'Energie Atomique.

(2) Ingénieur au Commissariat à l'Energie Atomique, Chargé de Mission au Centre de Formation Internationale à la Gestion des Ressources en Eau (CEFIGRE), Sophia Antipolis, BP 13, 06560 VALBONNE, FRANCE.

(3) Maitre Assistant an Laboratoire de Pharmacie Chimique et d'Hydrologie, UER des Sciences Pharmaceutiques et Biologiques de Grenoble, Université Scientifique et Médicale de Grenoble, France. 


\subsection{Les différentes approches d'une étude toxicologique simplifiée.}

Dans une étude toxicologique simplifiée, pour laquelle on se contentera de cerner l'action de la substance prise isolément et on ne prendra pas en considération les effets d'antagonismes et de synergies évoqués plus haut, on distingue, dans l'ordre de difficulté et de complexité croissantes, les différents niveaux d'approche présentẻs dans le tableau suivant:

\begin{tabular}{lll}
\hline Domaine & Type d'effet étudié & Support \\
\hline $\begin{array}{l}\text { Flore et animaux } \\
\text { inférieurs }\end{array}$ & $\begin{array}{l}\text { EFFETS } \\
\text { ECOLOGIQUES }\end{array}$ & $\begin{array}{l}\text { Daphnies } \\
\text { Escargots } \\
\text { Amphibiens }\end{array}$ \\
\hline & $\begin{array}{l}\text { TOXICITE AIGUE } \\
\text { (effet léthal) }\end{array}$ & \\
\cline { 2 - 3 } $\begin{array}{l}\text { Animaux supérieurs } \\
\text { et homme }\end{array}$ & $\begin{array}{l}\text { TOXICITE CHRONIQUE } \\
\text { (morbidité) }\end{array}$ & $\begin{array}{l}\text { Souris, } \\
\text { rats, cobaye, etc. }\end{array}$ \\
\cline { 2 - 2 } & $\begin{array}{l}\text { TOXICITE RETARDEE } \\
\text { OU DIFFEREE (à seuil) } \\
\text { (pouvoir carcinogénétique) }\end{array}$ & \\
\hline
\end{tabular}

\section{Remarques:}

1) Pour la flore et les animaux inférieurs, il n'existe en France qu'un seul test normalisé, c'est le test «Daphnies», les autres font l'objet de recherches ou de mises au point.

2) Il semble que le test «Daphnies» fournisse des résultats allant dans le même sens que les tests concernant les petits animaux et puisse être considéré comme un bon témoin des effets toxicologiques sur ces derniers.

\section{ETAT DES RECHERCHES BIBLIOGRAPHIQUES SUR LA TOXICITE DES COLORANTS FLUORESCENTS UTILISES COMME TRACEURS EN HYDROLOGIE.}

Les éléments épars de toxicologie ainsi que les bribes d'information réglementaires que nous avions réussi à recueillir depuis 1964, début de nos activités dans le domaine des traceurs fluorescents, viennent d'être heureusement et solidement complétés par trois études récentes: celle de Rochat/Demange/Rérat (1977) qui présente les travaux réalisés dans le cadre d'une thèse (Rérat 1976) uniquement consacrée à la Rhodamine $\mathrm{B}$, celle de Smart (1976) où figure une synthèse toxicologique détaillée sur les azurants optiques, et enfin celle de Smart et Laidlaw (1977) qui contient une synthèse, fort documentée et d'un 
intérêt primordial, sur la plupart des traceurs fluorescents utilisés en hydrologie.

Dans un souci d'exhaustivité, nous avons tenté de compléter cette abondante documentation par une recherche systématique effectuée par l'entremise de M. Braffmann, Chef du Service de Documentation Elf Solaize, et par les soins de Mme Paraire, du Service de Documentation Elf Paris-Tour Aquitaine, que nous tenons á remercier pour leur précieuse collaboration.

Cette derniére démarche, qui ne nous a permis de recenser que quelques travaux d'intérêt mineur, nous autorise à dresser l'état des connaissances à partir des seules études bibliographiques susmentionnées.

\section{TOXICITE DE LA RHODAMINE B UN EXEMPLE D'EVOLUTION DES CONNAISSANCES, DES RECHER- CHES ET DES REGLEMENTATIONS.}

\subsection{La prééminence de la Rhodamine B dans l'essor des traceurs fluorescents et dans l'état d'avancement des études toxicologiques relatives à leur utilisa- tion}

Lors des premiéres expériences de mise en oeuvre de la Rhodamine B, expériences qui ont révélé le grand intérêt de ce traceur et de quelques autres colorants de la famille du xanthène, la Rhodamine B figurait avec son «homologue» l'uranine qu'elle venait concurrencer, au tableau des colorants autorisés aux Etats Unis dans la préparation des médicaments et des cosmétiques sous les désignations respectives ( $\mathrm{D} \& \mathrm{C}=$ Drug an Cosmetic):

\begin{tabular}{lll}
\hline Nom commercial & Réf. COLOUR INDEX & $\begin{array}{l}\text { Désignation Food and Drug } \\
\text { Administration of U.S.A. }\end{array}$ \\
\hline Fluorescéine & $\begin{array}{l}\text { C.I. } 45350 \\
\text { Acid Yellow } 73\end{array}$ & D \& C Yellow $n^{\circ} 7$ \\
Uranine & $\begin{array}{l}\text { C.I. } 45350 \\
\text { Acid Yellow } 73\end{array}$ & D) \& C Yellow $n^{\circ} 8$ \\
Rhodamine B & $\begin{array}{l}\text { C.I. } 45170 \\
\text { Basic Violet } 10\end{array}$ & D) \& ( Red $n^{\circ} 19$ \\
\hline
\end{tabular}

[KIRK OTHNER (....), COLOUR INDEX (1956)]

Il semble que cette caution n'ait pas été jugée suffisante, à la fois par les expérimentateurs et par leurs commanditaires, assurément soucieux de rassurer le public sur les conséquences de ces colorations inattendues, puisque Smart et Laidlaw (1977) signalent que Pritchard et Carpentier avaient fait entrepren- 
dre, dés avant 1960, des tests sur des poissons dans le cadre de leurs études sur les estuaires soumis à marée.

Le fait que les études systématiques du pouvoir carcinogénique des substances organiques [Shubik, Hartwell (1969)] aient révélé l'activité de très nombreux composés, dont les rhodamines, n'est sans doute pas étranger á la multiplication des études toxicologiques et à des restrictions d'emploi dont on verra qu'elles relèvent d'une prudence extrême que l'on pourrait taxer d'excessive.

\subsection{Nature des travaux toxicologiques entrepris sur la Rhodamine B}

Ainsi que nous l'avons précédemment indiqué, les études bibliographiques de Smart et Laidlaw (1977) et de Rochat et All (1977) recensent la quasi totalité des travaux sur le sujet. Comme ces deux études se recouvrent peu, nous convions le lecteur désireux d'approfondir le sujet, à rechercher dans l'une et l'autre les commentaires sur les références susceptible de l'intéresser.

L'ensemble de ces travaux couvre un champ extrêmement vaste et une analyse succinte établit que tous les niveaux d'une étude toxicologique limitée à l'action de la substance prise isolément ont été étudiés.

Parker (1973) [Cité par Smart/Laidlaw (1977)] a même abordé une étude de synergie révélant l'effet aggravateur de la Rhodamine B sur l'Antimycine A. On doit toutefois noter que, quel que soit le réactif biologique [animaux inférieurs (depuis les tests sur daphnies jusqu' aux études des effets sur les poissons et amphibiens en passant par les mollusques) ou animaux supérieurs (rats ct souris)], la plupart des auteurs n'ont obtenu des effets marqués que pour de très fortes concentrations en Rhodamine B, toujours au moins 100 fois supéricures aux niveaux de concentration provoquant une coloration de l'eau.

\subsection{Résultats de ces travaux}

Sauf rares exception, les tests toxicologiques n'ont pas été restreints à la seule Rhodamine B. Les premiéres études ne prennent que l'uranine (fluorescéine sodique) pour terme de comparaison, mais les plus récentes se sont étendues à la gamme des traceurs xanthéniques et même aux autres traceurs dont le développement est envisagé (notamment les azurants optiques).

("est assurément cette confrontation qui est á l'origine du discrédit qui semble frapper la Rhodamine B.

In effet il est apparu à de nombreux auteurs, cités par Smart et Laidlaw (1977), que la Rhodamine B (et accessoirement la Sulforhodamine B) présentait, dans les conditions choisies, les plus grands risques de toxicité; une des catuses en serait, selon little et Lamb (1973) [cités par Smart et Laidlaw (1977)] la forme cationique de cette substance qui en faciliterait la fixation sur les tissus vivants.

A ecei sajoute le fait que les études de sapidité d'canu potables ont montré 
que, lorsque ces eaux sont chlorées d'assez faibles concentrations en Rhodamine $\mathrm{B}$, de l'ordre de $10^{-8}$, provoquent la formation de chlorophénols en teneurs suffisantes pour altérer le goût des eaux de boisson [Wilson (1968) cité par Smart et Laidlaw (1977)].

\subsection{Incidence sur l'utilisation de la Rhodamine B en hydrologie.}

Le retrait en 1960 de la Rhodamine B de la liste des substances autorisées dans l'industrie des médicaments et des cosmétiques par la Food and Drug Administration des USA [Colour Index, Supplément á la Seconde Edition (1963) p.s 233], ainsi qu'une interprétation pessimiste des résultats de certains travaux toxicologiques a donné lieu aux Etat Unis à des mesures de caractère alarmiste (1). Faute de pouvoir prendre connaissance des documents (2) cités par la même source d'information [Bakalowicz (1973, Communication privée)], nous avions consulté, en juin 1973, l'United States Environmental Protection Agency des USA dont le responsable des études de protection des eaux nous avait précisé qu'à cette date aucune limite, autre que celle s'appliquant à toute substance organique, n'avait été fixée pour la Rhodamine B.

Cette information, en apparente contradiction avec l'avis plus haut précité, semble devoir ramener à de plus justes proportions les risques afférents à l'utilisation de ce traceur.

D'ailleurs, Smart et Laidlaw (1977), ainsi que Rochat et All (1977), lesquels ont eux mêmes procédé à des études toxicologiques, s'accordent sur le fait que,tous les résultats positifs ayant été obtenus à doses très élevées, il ne semble pas que la présence de caractère occasionnel ou accidentel de Rhodamine B dans les eaux aux concentrations usuellement pratiquées dans des opérations de traçage en hydrologie, puisse présenter un risque immédiat pour le milieu.

\section{ETAT DES CONNAISSANCES TOXICOLOGIQUES ET DE. LA RE(;I.F- MENTATION RELATIVE A L'USAGE DE TRACEURS FLUORES( ENTS EN HYDROLOGIE [SMART ET LAIDLAW (1977)]}

Nous ne pouvons faire mieux que reprendre ici l'essentiel des conclusions sur

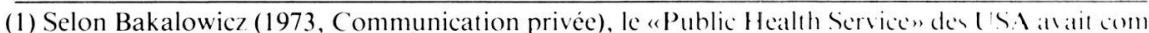
muniqué dans la revue spéléologique américaine N.S.S. New (n 7. 1966. P. 179) un ani cilc dam Spelunca Bulletin, Revue de la Fédération Française de Spéleologic, anni libclli:

«Do not use Rhodamine B dye!

The Public Healt Service advises that Rhodamine B dye - frequents used for stram trating hats been found to have certain carcinogenic (cancer producing properties). Ihe resulte of kest ate comsi dered sufficient justification by the federal Water Pollution Contod Administration to discontinte the use of Rhodamine $B$ dye, (avers are requested not fo use this tratcing ateent...

(2) Autres documents signales par Bakalowic, (1973, (ommunication prisce)

a) Safe parameters for Rhodamine $B$ dye.

N.S.S. News n 9.1966. pp 2()4-2()5

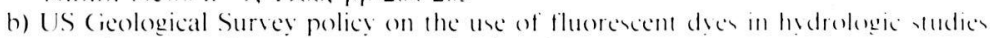

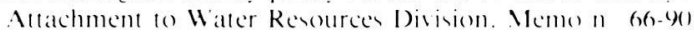


ce point de la remarquable synthèse de Smart et Laidlaw (1977) consacréc á l'étude comparée des traceurs suivants:

Rhodamine B

Rhodamine Wt

Sulforhodamine B

Uranine
Lissamine

Pyranine

Amino $\mathrm{G}$ acide

Photine CU

\subsection{Toxicologie comparée des traceurs fluorescents}

Il ressort des diverses études toxicologiques examinées par Smart et Laidlaw (1977) que la Rhodamine Wt, la fluorescéine et la Photine CU présentent des risques de toxicité relativement faibles, alors que ceux de la Rhodamine B et de la Sulforhodamine B semblent comparativement plus importants.

En l'absence d'autres données sur ce produit, Smart et Laidlaw (op. cité) font état de déclarations des fabricants assurant la problable inocuité de la Lissamine FF.

\subsection{Réglementations sur l'usage des traceurs fluorescents}

L'Uranine et la Pyranine figurent sur la liste des substances autorisées dans l'industrie des médicaments et des cosmétiques par la Food and Drug Administration des USA tandis que la Rhodamine B en a été retirée en 1960 ( $\mathrm{cf}$ 3.4.). La Rhodamine B et l'Uranine ont été classées dans la catégorie toxicologique C 111 par les organisations mondiales F.A.O. et O.M.S.

Aux Etats Unis, l'utilisation de la Rhodamine B dans des eaux susceptibles d'alimenter des réseaux de distribution d'eau potable est généralement évitée. Les quantités maximales permissibles d'ingestion ont été fixées, pour la Rhodamine B. la Sulforhodamine B et la Rhodamine $\mathrm{Wt}$, à $0,75 \mathrm{mg} / \mathrm{jour}$, quantités qui peuvent éventuellement être dépassées si la concentration d'ingestion reste inférieure à $370 \mu \mathrm{g} / \mathrm{l}\left(3,710^{-7}\right)$.

Enfin, l'US Geological Survey recommande que la mise en oeuve des raceurs ne conduise pas à des concentrations finales supéricures à $10 \mu \mathrm{g} / 1\left(10^{4}\right)$.

\section{CONCLUSIONS}

On ne peut, comme Smart et Laidlaw (1977), que recommander, en l'absence de données plus précises, l'adoption de normes analogues à celles qui semblent devoir être adoptées aux Etats Unis et qui paraissent prémunir contre tout risque d'atteinte grave à l'équilibre écologique du milieu naturel.

Le respect de ces niveaux de concentration, dont on sait qu'ils ne sont aucunement contraignants pour la mesure, aurait d'ailleurs pour avantage d'éviter la contamination de sites (sur le plan traçage s'entend).

La seconde recommandation, qui rejoint la premiére dans ses objectifs, consi- 
ste en l'instauration d'une certaine discipline parmi les utilisateurs de traceurs que l'on doit convier à soumettre leurs projets à l'approbation d'organismes de concertation capables de juger à la fois de leur opportunité et de leur compatibilité. [Molinari (1977)].

Il resterait bien entendu à inciter la poursuite d'investigations plus poussées et à imposer, avant toute mise en oeuvre de nouveaux traceurs, l'exécution de recherches toxixologiques. C'est ce que prévoit d'ailleurs la nouvelle réglementation française sur le contrôle des produits chimiques (loi du 12 juilett 1977; décret du 15 janvier 1979) dont l'entrée en vigueur est fixée au 1 juillet 1979.

\section{RÉSUMÉ}

Cette synthèse a été établie en juin 1977 à partir de trois etudes très récentes et d'une recherche bibliographique complémentaire.

Elle couvre l'ensemble des substances fluorescentes actuellement utilisées ou expérimentées comme traceurs en hydrologie et attire l'attention sur les usages et la réglementation relatifs à leur mise en oeuvre.

En annexe est jointe une liste exhaustive des publications recensées sur le sujet (35 titres).

\section{BIBLIOGRAPHIE}

\section{A. ETUDES ET OUVRAGES CITES}

COLOUR INDEX, Second Edition (1956) in Four Volumes. The Society of Dyers and colourists, Bradford, Yorkshire, England. The American Association of Textile Chemists and Colourists Lowell, Massachussets, USA.

COLOUR INDEX, Second Edition - Supplement (1963). The Society of Dvers and Colourists, Bradford, Yorkshire, England.

KIRK OTHNER: Encyclopedia of Chemical Technology. Second Edition, Vol 22, Xanthene Dyes pp. 433-437

MOLINARI J.: Perspectives offertes par l'utilisation rationnelle des traceurs naturels et artificiels en hydrologie karstique. Commentaires de nombreux exemples récents de multitraçages. Deuxième Colloque d'Hydrologie en Pays Calcaire. Annales Scientifiques de L. 'Université de' Besançon (1976), Géologie, Fascicule 25, 3éme série. pp. 275-306.

RERAT J. C1.: La Rhodamine B - Propriétés physicochimiques - Etude de leurs incidences sur son utilisation comme traceur en hydrologie et sur son épuration dans les effluents. Thesese de loctorat en Pharmacie - Université Scientifique ct Médicale de Grenoble (1976), Octobre, 132 pages. SHUBIK P./ HARTWELL J.L.: Survey of compounds which have been tested for carcingenic activity. Supplement 2 (1969), pp. 533 - 534. US Department of Health, Edlucation and Welfure Public Health Service. J.À. Peters Ed., National cancer Institute, Bethesda. nd. ISSA.

SMART P.L.: The use of optical brighteners for water tracing. Truns. British Cave Reseurch 4 sociation (1976) Juillet, 3(2), pp. 62-76.

SMART P.L./LAIDLAW I.M.S.: An evaluation of some fluorescent dyes for water tracing. W a ter Resources Research (1977) Février, 13 (1), pp. 15-33.

ROCHAT J/DEMENGE P/RERAT J. C1: Contribution à l'etude toxicologique d'un traceur fluorescent: la Rhodamine B. Toxicological European Research (1978),1, pp. 23-26.

\section{B.AUTRES REFERENCES}

NOTA: A fin de faciliter les recherches, cen references ont ede preciden den signes unisante precisant leur origine:

(M): notre propre bibliographic (Nai 1977)

(R): RO(HAT et All. (1977) [Rhodamine B]

(S): SMART (1976) [azurant optiques] 
(SI ): SMART/IAII)I AW (1977)

(S) - (SL) AKAMATSU K./MATSUO M. - Safety of optical whitening agents (en Japonais). Senryo To Yakuhin (1973) 18 (2), pp 2-11. Traduction en Anglais: Trans Programme RTS 9415 -Brit. Libr. Boston Spa. Yorkshire, England, Juin 1975.

(SL) -BANDT H.J. - Giftig oder ungiftig für Fische? Deut. Fish. Rundsch. (1957) 4 (6), pp 170171

(S) - CARTER R.O. - Safety of fluorescent whitening agents in detergents. Proc. Symp. Fluorescent Whitening Agents. Stockholm (1973) Avril, MVC Report 2.

(R) - DEROUAUX G./ LECOMTE J. - Dérivés du triphénylméthane et muscle strié d'amphibien. C.R. Séances Soc. Biol. (1950), 144, pp 1713-1714

(M) - MC DONALD T./ KASTEN K./ HERVEY R./ GREGG S./ ROBB C.A./ BORGMANN A.R. - Acute and subacute toxicity evaluation of intravenous sodium fluorescein in mice rats and dogs. Toxicol. Appl. Pharmacol. (1974), 29 (1), pp. 97-98

(M) - GANGOLLI S.D./ GRASSO P./ GOLDBERG L./ HOOSON J. - Protein binding by food colorings in relation to the production of sub cutaneous sarcoma. Food Cosmet. Toxicol. (1972), $10(4)$, pp 449-462

(SI.) -GANZ C.R./ SCHULZE J./ STENSBY P.S. - Accumulation and elimination studies of four detergent fluorescent whitening agents in bluegill (Lepomis Macrochirus). Environm. Si.i. Technol. (1975), 8 (8), pp 738-744

(S) - (il OVER R.R. - Optical brighteners A new tracing reagent. Trans. Cave Res. Grp. GB (1972), $14(2)$, pp. 84-88

(S) - (iLOXHUBER C./ HECHT G./ KIMMERLE G. - Toxikologische Untersuchungen mit Aufhellern (Blankophor-Typen). Archiv für Toxikol. (1962), 19, pp 302-312

(SI ) - HANSEN W.H./ FITZHUGH O.G./ WILLIAMS M.A. - Subacute oral toxicity of nine D and $C^{\prime}$ coal tar colours. Jr. Pharmacol. Exp. Ther. (1958), 122, 29 A

(S) - HESS R. - Experimental toxicology of compounds used as optical brighteners. Proc. Symp. Fluorescent Whitening Agents. Stockholm (1973) Avril, MVC Report 2.

(S) - (SI ) - JENSEN S./ PETTERSSON O. - 2,5, di-(benzoxazole-2-yl) thiopine, an optical brightener contaminating sludge and fish. Environm. Pollut. (1971), 2, pp 145-155

(R) - KATASI MAKINO/MASURU MASAYAMA - The dye that cause specific heart muscle degeneration. Jr. Oriental Med. (1939), 29, pp 1231-1233. in Chemical Abstracts (1939), 33. 3075 .

(S) - (SI ) - KEPLINGER M.L./FANCHER O.E./LYMAN F.L./CALANDRA J.C. - Toxicological studies of four fluorescent whitening agents. Toxicol. Appl. Pharmacol. (1974). 27, pp 494-504.

(1) - KHANNA S.K./ SINGH G.B. - Toxicity of commonly used food colours. A review. Jr. Scientific and Industrial Research. (1975) Nov., 34 (11), pp 631-636

(S) - KORTE F. - Ecological aspects of fluorescent whitening agents. Proc. Symp. Fluorescent Whitening Agents. Stockholm (1973), Avril, MVC Report 2.

(S) - (SI ) - I ITTIE I..W. LAMB J.C. - Acute toxicity of 46 selected dyes to Fathead minnow (Pimephales Promelas). Dyes and Environment, Vol 1, chap. 5. American Dye Manufacturers Institute, New York (1973)

(SI) - MARKIN( I I.L.. - Toxicity of Rhodamine B and fluorescein sodium to fish and their compatibility with Antimycin A. Prog. Fish. Cult. (1969), 31, pp 139-142

(R) - MIC HI I SON F.H. - Schell fluorescence in Australorbis Glabratus and other aquatic snails exposed to tetracyclines. J. Parasitology (1964) 50, pp 743-747.

(S) - NI. I KOMM S. DF: TREY M. - Etude de certains azureurs optiques du point de vue de leur pouvoir cancerigence el co-cancérigéne. Med.Exp. (1961), 4, pp 298-306.

(SI) - PNCIIRA M. - Toxicing of Rhodamine B to eggs and larvae of Crassotrea Virginica. Proc. Vat. Shellfish. Ass. (1967), 58, pp 7-8.

(R) - (S) - PARKLR (i.Ci.Jr. - Tests of Rhodamine Wt dye for toxicity to oysters and fish. J. Res. l'.S. (ieol. Surt: (1973), 1, p 499.

(SI) -PRIT(HARI) I). W'. (ARPINTER J.H. - Measurement of turbulent diffusion in estuarinc and inhore wallers. Bull. Imt. Ass. Sici. Hidrol. (196()), 20, pp 37-50.

(S) SNYIAR I.H. (OPI)YKF I).I RUBFNKFONIC H.I. - Toxicological studies on brightonces. Toxicol. Applied Pharmacol. (1963). 5. pp 176-183.

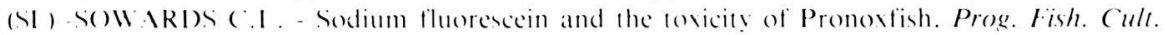


(1958), 20 (1), p 20.

(S) - STENSBY P.S. - Optical brighteners and their evaluation. Soap Chem. Spec. (1967), Avril, Mai, Juillet, Septembre; 43,41, 84, 80, 94, 96.

(S) - (SL) - STURM R.M./ WILLIAMS K.E./ MACEK K.J. - Fluorescent whitening agents. Acute fish toxicing and accumulation studies. Water Research (1975), 9, pp 211-219.

(SL) -UMEDA M. - Experimental study of xanthene dyes as carcinogenic agents. Gann. (1956), 47, pp 51-78.

(R) - WATARU KUBOKAWA - Hemolytic action of toxic dyestuffs in food. Kyoto Furitsu Ika Daigaku Zasshi (1960), 68, pp 286-288 et 337-340. in Chemical Abstracts. (1961), 55, pp 4794.

(SL) -WEBB J.M./ FONDA M./ BROUWER E.A. - Metabolism and excretion patterns of fluorescein and certain halogenated fluorescein dyes in rats. J. Pharmacol. Exp. Ther. (1962), 137 (2), pp 141-147.

(SL) -WEBB J.M./ HANSEN W.H. - Studies of the metabolism of Rhodamine B. Toxicol , Appl. Pharmacol. (1961), 3 (1), pp 86-95.

(R) - (SL) - WEBB J.M./ HANSEN W.H./ DESMOND A./ FITZUG O.G. - Biochemical and toxicologic studies of Rhodamine B and 3,6, diamino-fluoran. Toxicol. Appl. Pharmacol. (1961), 3, pp 693-706.

(SL) -WILSON J.F. - Fluorimetric procedures for dye tracing. Techniques of Water Resources Investigation of the U.S. Geological Survey, Vol 3, 31 pages, U.S. Geological Survey, Washington D.C. (1968).

(SL) -WOELKE C.E. - Development of a receiving water quality bioassay criterion based on the $48 \mathrm{hr}$. Pacific oyster (Crassostrea Gigas) embryo. Techn. Report. 9 - Wash. Dept. Fish. (1972).

(S) - ZINKERNAGEL R. - Fluorescent whitening agents and the water system. Proc. Symp. Fluorescent Whitening Agents. Stockholm (1973) Avril, MCV Report 2. 